surface. Can such a tetrad vary while its points remain upon a twisted cubic curve? Professor White showed that while this is not possible for every cubic and quadric, it can occur for special quadrics invariantively conditioned by the cubic curves. Given the curve and two polar tetrads upon it, the equation of the surface was explicitly determined, a surface triply tangent to the curve. The problem was extended to polar pentads of a cubic surface, to forms of all orders above the first in 4space, and to cubics, quartics, etc., in the plane, the vertices moving always upon the rational norm-curve of the space under consideration.

Evanston, February 4, 1905.
Thomas F. Holgate, Secretary of the Section.

\title{
MATHEMATICS AT THE ST. LOUIS CONGRESS, SEPTEMBER 20, 22, AND 24, 1904.
}

Is the scheme of the Congress of Arts and Science connected with the Louisiana Purchase exposition, mathematics was classified as a department under the division of normative science, philosophy being the other department of that division. At 10 o'clock on Tuesday, September 20, both departments met and listened to the divisional address entitled "The science of the ideal," by Professor Josiah Royce, of Harvard University. An abstract of a paper itself so condensed as this is hardly a possibility; the paper has been published in Science (October 7, 1904).

Immediately following this joint session was the opening session of the department of mathematics, later to be subdivided into three sections. The officers of this session were Professor H. S. White, chairman, and Professor G. A. Bliss, secretary. Two addresses had been provided for this session, both of a highly general character, as distinguished from the more special discussions of sectional meetings. The first, by Professor Maxime Bôcher of Harvard University, was upon "The fundamental conceptions and methods of mathematics"; the second, by Professor James Pierpont of Yale University, on "The history of mathematics in the nineteenth century." Both have appeared already in this Bulcetin.* The auditors at this, as

* This volume, pages 115-135 and pages 136-159 respectively. 
at the sectional meetings, numbered between 60 and 70 .

On Thursday, September 22, the section of Algebra and Analysis met at 10 o'clock in Hall 1 of the library building, with Professor E. H. Moore as chairman and Professor G. A. Bliss as secretary. The two principal addresses were by Professor Emile Picard of Paris, entitled "Sur le développement de l'analyse mathématique et ses rapports avec quelques autres sciences," and by Professor Heinrich Maschke of Chicago, on "The present problems of algebra and analysis." The former has appeared in the Bulletin des sciences mathématiques for October and November, 1904, and will appear in an authorized translation in this BULLETIN within a short time.* The latter was an extended survey of the present state of the theory of invariants of quadratic differential forms in $n$ independent variables, an intensive study of differential parameters or the Biegungsinvarianten of surfaces, and included a resumé of the author's papers in the Transactions of this Society, Volumes 1 and 4.

Under the order of "Shorter Communications" the following papers were presented :

(1) E. V. Huntington : "A set of postulates and axioms for real algebra."

(2) J. I. Hutchinson: "On the uniformizing of algebraic functions."

(3) M. B. Porter: "On functions defined by an infinite series of analytic functions of a complex variable."

(4) E. R. HEDRICK : "On the generalization of the analytic functions of a complex variable."

(5) M. W. HASKELL : "On collineations in ordinary space."

(6) J. B. SHAw : "On linear associative algebra."

(7) G. A. MILleR : "The bearing of several recent theorems in group theory."

In the absence of authors, No. 3 was read by Professor Bôcher, No. 7 by Professor Pierpont.

On Saturday at 10 o'clock, the section of Geometry met in the same hall, with Professor Thomas F. Holgate as secretary. In the absence of the chairman appointed by the directors of the congress, Professor W. E. Story, the section elected Professor M. W. Haskell as chairman. The two chief addresses

*A translation by Professor G. B. Halsted appeared in Science, December 23, 1904. 
were by M. Gaston Darboux of Paris, "Etude sur le développement des méthodes géométriques," and by Dr. Edward Kasner of Columbia University: "The present problems of geometry." The former was published in the Bulletin des sciences mathématiques for September, 1904.* Dr. Kasner's address appeared in the March number of the Bulletin.

At the close of the principal addresses, shorter communications were presented by the following:

(8) H. F. BLICHFELDT : "Concerning some geometrical properties of surfaces of revolution."

(9) G. A. Buiss: "The geometry of Jacobi's criterion for the problem of the calculus of variations involving several unknown functions."

(10) L. W. DowLING: "On the generation of certain unicursal plane curves."

(11) Arnold EMCH : "The configuration of the points of inflexion of a plane cubic and their harmonic polars."

(12) G. B. Halsted : "Non-euclidean spherics."

(13) H. HANCOCK : " Algebraic minimal surfaces."

(14) H. P. Manning: "Representation of complex variables in space of four dimensions."

The third section, Applied Mathematics, met at 4 P. M. on Saturday in the gymnasium, with Professor A. G. Webster as chairman and Professor H. T. Eddy as secretary. The first address was on "The rôle of hypothesis in modern exact science", by Professor Ludwig Boltzmann, of Vienna; the second by Professor H. Poincaré, of Paris, on "The principles of physics." The latter has appeared, translated by Professor G. B. Halsted, in the Monist for January, 1905. Following these longer papers, Professor James McMahon presented a communication: "On the use of $n$-fold Riemann spaces in applied mathematics."

Aside from these strictly mathematical sessions, many meetings of cognate departments attracted hardly a less number of our members, and the publication of the collected papers from all departments will be eagerly awaited as the necessary adjunct and supplement to the oral proceedings of the Congress. One purpose of the directors of the Congress was certainly realized

\footnotetext{
* A translation by Professor Halsted appeared in the Popular Science Monthly, March, 1905.
} 
- the conceptions of science as an organic whole, and of the community of interest, the necessary interdependence, of all special divisions of science were fitly expressed and strikingly enforced by this unique gathering. No narrow notion of a scientist's calling could survive for a day in that high atmosphere.

Brief outlines of the above papers, so far as they could be obtained, are given here. Papers already published are omitted. Numbers correspond to those in the lists above.

1. Dr. Huntington's paper has appeared in full in the Transactions for January, 1905.

3. Professor Osgood established in the Annals of Mathematics, volume 3 (1901), page 25, an important theorem on functions defined by a series of analytic functions. Professor Porter generalizes it by removing the condition that the point set shall be everywhere dense in the region $T$ of convergence, and substitutes the condition that the series shall converge for all values of the argument pertaining to a point set whose limiting points are everywhere dense on a closed rectifiable contour $C$ lying inside the region $T$.

4. Professor Hedrick proposed to generalize the notion of function $u+i v$ of a complex variable $x+i y$ by requiring the real and imaginary parts, $u$ and $v$, to satisfy not the Laplacian, but a more general form of linear differential equation of the second order. A specially interesting feature of these generalized functions would be the possibility that they might give a depiction of the $(x, y)$-plane conformal in the sense of an elliptic or hyperbolic geometry.

5. Professor Haskell announced several theorems on the resolution of collineations into generators of special type, and in particular on periodic collineations in space of $n$ dimensions.

6. Professor Shaw presented a topical outline of an extensive treatise, in which it is purposed to set forth the present state of linear associative algebra, not in a chronological or comparative study of the different forms of known algebras, nor in the exhaustive study of any one form, but in the tracing of the general laws of the whole subject. The paper is in three parts : 
general theory, particular systems, and applications. The first part is thoroughly analyzed, consisting of twelve chapters developing the theory fully and independently, with a thirteenth which discusses the linear associative algebra as related to other algebras and to general algebra; of the second and third parts only a general description is given.

7. Professor Miller's short review referred mainly to enumerations of subgroups in groups of special type. A new theorem was the following. If a group of order $p^{m}, p$ being any prime, contains more than one cyclic subgroup of order $p^{\beta}>4$, it contains a multiple of $p$ such subgroups, where $\beta$ again can have any value from 2 to $m-1$.

8. If 4 or more points on any surface be taken, relations will subsist between their geodesic distances and the angles of the geodesics. Professor Blichfeldt proposes to exclude the angles from consideration, and inquire whether relations subsist among the distances. For more than 5 points, there will always be relations of this kind. For 5 points, if the surface be one of revolution, there will be one such relation. The author is not certain that the only surfaces having this property are those of revolution, but has proved that if two or more relations subsist among the 10 geodesic distances of 5 points on a surface, the surface has constant curvature.

10. In a net of plane curves with fixed fundamental points, the term involution curve is defined according to Castelnuovo. If an involution curve is composed of two algebraic curves, the two parts are termed conjugate in the involution defined by the net. Professor Dowling establishes certain general classes of degenerate involution curves having a line or a conic as one part, and so has a method of generating the conjugate part. This method he applies in particular to involutions of the second order, and obtains the the following three classes of unicursal curves : (a) a curve of order $2 n-1$ with multiple points indicated by $1_{2 n-5},(n-3)_{4}, 6_{2} ;(b)$ a curve of order $2 n-2$ with multiple points indicated by $4_{n-2},((n-3) / 2)_{4}, 3_{2} ;(c)$ a curve of order $2 n-2$ with multiple points indicated by $2_{n-1}, 2_{n-3}$, $((n-4) / 2)_{4}, 4_{2}$.

Using involutions of order higher than 2, curves usually not unicursal can be found as conjugates to particular lines and 
conics. The author states for example that every plane quintic can be regarded as conjugate to a line in an involution determined by a net of cubics, having a suitable number of fundamental points.

11. By the aid of parametric representation of the points on a non-singular plane cubic, Professor Emch discusses the collinearity of the 27 points of contact of tangents to the cubic from its 9 inflexions. He finds 81 lines containing each 3 such points, and 81 other lines connecting 2 contact points with an inflexion.

12. Professor Halsted gave a brief outline of "intrinsic spherics", or spherical trigonometry independent of the parallel postulate, and advocated from a pedagogical standpoint the introduction of the sphere as two-dimensional figure into elementary geometry, instead of the use of the three-dimensional globe.

13. The problem of determining all algebraic minimal surfaces was solved analytically by Weierstrass in 1866 (see his collected works, volume 3, pages 39-52). Darboux, in his Théorie générale des surfaces, part 1, No. 221, has shown that such surfaces can always be generated by the translation of an algebraic curve. This shows that the function $F(s)$ used in the formulas of Weierstrass must be algebraic. Professor Hancock proposes as a problem next to be attacked the question of determining all those minimal surfaces which are not themselves algebraic, but contain a sheaf of algebraic curves.

Evanston, ILI.,

H. S. White.

March, 1905.

\section{ON THE USE OF HYPERCOMPLEX NUMBERS IN CERTAIN PROBLEMS OF THE MODULAR GROUP.}

BY DR. J. W. YOUNG.

(Read before the American Mathematical Society, September 17, 1904.)

THE following discussion connects two subjects which have hitherto been considered apart, and indicates a method of attacking certain modular group problems which seems susceptible of further elaboration. 